\title{
Editorial: Moving an Interdisciplinary Journal Forward
}

I am honored and thrilled to assume the position of Lead Editor for Physical Review E. PRE is a truly interdisciplinary journal whose scope extends well beyond traditional physics to include many areas of science and engineering. While the set of topics covered by PRE is broad, they are all rooted in the behavior of complex interacting many-body systems, typically subject to nonlinear, often stochastic, collective dynamics. These systems are frequently (but not exclusively) in the classical realm, and their analytical, numerical, and experimental exploration tends to rely on statistical approaches.

This diversity of research creates exciting opportunities and challenges, as many of the subfields covered by PRE — and the boundaries between them-evolve swiftly. We must therefore be nimble in how we define the journal's scope and its sections and in how we populate our Editorial Board with the right expertise. Our editors must also continually interact with the scientific community to keep the journal topical and to strengthen coverage of emerging or intensifying research areas.

As I step into this new role, I would like to recognize my predecessor Eli Ben-Naim, who directed PRE with expert, steadfast, and thoughtful leadership for more than eight years. Building on the foundations laid by the journal's first two Lead Editors, Irwin Oppenheim and Gary Grest, Eli continued to expand PRE's interdisciplinary scope while maintaining the journal's renown and premier quality. Under his tenure, the journal introduced Editors' Suggestions, a collection of "Milestones" for the journal's 25th anniversary, and "Spotlights" - highlighted articles that showcase a topical area, such as the physics of climate or of laser-created pair plasmas. He also helped establish the annual Irwin Oppenheim award, which recognizes outstanding contributions to physics by early career scientists who publish in PRE. (The first award was presented at the 2019 APS March Meeting.) On behalf of PRE's entire editorial team and the scientific community it serves, I thank Eli for his commitment and dedication.

I look forward to collaborating with the journal's exceptional, dedicated staff and Editorial Board as well as with the APS leadership to maintain PRE's eminent quality and reputation. We expect papers published in PRE to make a significant contribution in a specific research area, to generate interest for our readership, and to explore the addressed subject matter comprehensively and thoroughly. With our excellent authors and eminent pool of conscientious, knowledgeable, diligent, and timely referees, I am confident that we will succeed in further enhancing PRE's scientific standing and global reach. 